\title{
gs \\ Role of nonlocal exchange in the electronic structure of correlated oxides
}

\author{
Federico Iori, ${ }^{1}$ Matteo Gatti, ${ }^{1}$ and Angel Rubio ${ }^{1,2}$ \\ ${ }^{1}$ Nano-Bio Spectroscopy Group and ETSF Scientific Development Centre, Dpto. Física de Materiales, Universidad del País Vasco UPVIEHU, \\ Centro de Física de Materiales CSIC-UPVIEHU MPC and DIPC, Av. Tolosa 72, E-20018 San Sebastián, Spain \\ ${ }^{2}$ Fritz-Haber-Institut der Max-Planck-Gesellschaft, Theory Department, Faradayweg 4-6, D-14195 Berlin-Dahlem, Germany
}

(Received 19 December 2011; published 28 March 2012)

\begin{abstract}
We present a systematic study of the electronic structure of several prototypical correlated transition-metal oxides: $\mathrm{VO}_{2}, \mathrm{~V}_{2} \mathrm{O}_{3}, \mathrm{Ti}_{2} \mathrm{O}_{3}, \mathrm{LaTiO}_{3}$, and $\mathrm{YTiO}_{3}$. In all these materials, in the low-temperature insulating phases the local and semilocal density approximations (LDA and GGA, respectively) of density-functional theory yield a metallic Kohn-Sham band structure. Here we show that, without invoking strong-correlation effects, the role of nonlocal exchange is essential to cure the LDA/GGA delocalization error and provide a band-structure description of the electronic properties in qualitative agreement with the experimental photoemission results. To this end, we make use of hybrid functionals that mix a portion of nonlocal Fock exchange with the local LDA exchange-correlation potential. Finally, we discuss the advantages and the shortcomings of using hybrid functionals for correlated transition-metal oxides.
\end{abstract}

DOI: 10.1103/PhysRevB.85.115129

PACS number(s): 71.20.-b, 71.30.+h, 71.15.-m

\section{INTRODUCTION}

Nowadays, the standard model of electronic structure calculations is based on density-functional theory (DFT) in the Kohn-Sham (KS) formalism. ${ }^{1}$ The DFT-KS scheme, also in its simplest approximations like the local-density approximation $(\mathrm{LDA})^{1}$ or the generalized-gradient approximation (GGA), ${ }^{2}$ is generally highly successful in a very large variety of applications. Thus, when a LDA (or GGA) KS band structure turns out to be metallic in an insulating compound (e.g., in a transition-metal oxide), the result is often interpreted as a direct indication for strong electron correlation effects in the material, and a failure of the band-structure picture. A possible strategy that has been followed in the literature to overcome these difficulties is to resort to model approaches, like the multiband Hubbard model. In the LDA + U approach, ${ }^{3}$ LDA band structures are supplemented by an on-site Coulomb interaction (the Hubbard U) acting only on the "correlated" subset of the electronic degrees of freedom. In a higher level of theory, dynamical mean-field theory (DMFT), ${ }^{4}$ the Hubbard model is further mapped onto an Anderson impurity model, which can then be solved with different techniques, allowing for the description of dynamical effects beyond the LDA $+\mathrm{U}$. In those cases the LDA is claimed to be inadequate to capture the strong interactions taking place between correlated electrons in partially filled $d$ (or $f$ ) shells, which give rise to narrow bands in the solid.

However, we remark that DFT is a ground-state theory and $\mathrm{KS}$ band structures are not meant to describe the electronic excitations measured in photoemission, which also define the fundamental band gap of an insulator. Moreover, the LDA lacks the discontinuity of the local $\mathrm{KS}$ exchange-correlation (xc) potential $V_{\mathrm{xc}}(r)$ on a change of the electron number ${ }^{5-7}$ and suffers from a severe delocalization error, ${ }^{8}$ which is particularly relevant for localized $d$ and $f$ electrons. The underestimation of the fundamental band gaps in $s p$ semiconductors is well known,, 10 and understood in terms of self-energy corrections at the GW level of approximation. ${ }^{11,12}$ This underestimation sometimes may lead to metallic band structures also in "weakly correlated" small-gap semiconductors, like in germanium.

Here we consider several prototypical correlated transitionmetal oxides that have been studied by other methods in the past years: $\mathrm{VO}_{2}, \mathrm{~V}_{2} \mathrm{O}_{3}, \mathrm{Ti}_{2} \mathrm{O}_{3}, \mathrm{LaTiO}_{3}$, and $\mathrm{YTiO}_{3}$. In all the low-temperature insulating phases of these materials, the KS-LDA yields a metallic band structure. We thus address the following question: Is this just a result of the inadequacy of the LDA to deal with strong correlations or, rather, is this finding related to the systematic KS-LDA underestimation of band gaps that occurs also in weakly correlated semiconductors and gets enhanced in the oxides? To answer this question we make use of a generalized Kohn-Sham (gKS) scheme, ${ }^{13}$ where the local KS xc potential $V_{\mathrm{xc}}(r)$ is replaced by a spatially nonlocal $V_{\mathrm{xc}}\left(r, r^{\prime}\right)$. In these hybrid functionals, the nonlocal Fock exchange potential is mixed with the local (LDA or GGA) KS xc potential. ${ }^{14-16}$ In these approaches, the difference with LDA results stems solely from the nonlocal exchange term. This is not supposed to improve the description of electronic correlation. Therefore, if strong correlations are responsible for invalidating the KS-LDA description of these insulators, then one should expect to find the same problems in the gKS scheme. We will instead show how, without invoking strongcorrelation effects, the role of nonlocal exchange is essential to cure most of the LDA delocalization error and provide a band-structure description of the electronic properties of several transition-metal oxides in qualitative agreement with the experimental results (the same conclusion was reached for instance in the series of transition metal monoxides in Ref. 17).

The paper is organized as follows. In Sec. II we briefly introduce the generalized Kohn-Sham (gKS) scheme and the hybrid-functional parametrization that we use, and compare them with standard methods that treat electronic correlations. In Sec. III we present and discuss the results that we have obtained for the various transition-metal oxides, also across their metal-insulator phase transitions (MIT). ${ }^{18}$ Finally, in Sec. IV we draw our conclusions on the basis of these results and discuss the advantages and shortcomings of the 
use of hybrid functionals in the description of the electronic properties of correlated oxides.

\section{METHOD}

In the gKS scheme the xc potential is generalized to be nonlocal in space, ${ }^{13}$ contrary to the ordinary KS where it is local. In this framework, a common choice is to write the $\mathrm{gKS}$ nonlocal potential $V_{\mathrm{xc}}\left(r, r^{\prime}\right)$ as a sum of a nonlocal exchange term and a local KS correlation potential: ${ }^{14} V_{\mathrm{xc}}\left(r, r^{\prime}\right)=V_{x}\left(r, r^{\prime}\right)+V_{c}(r)$. For $V_{c}(r)$ a LDA or GGA expression is adopted, while the nonlocal exchange term $V_{x}\left(r, r^{\prime}\right)$ is obtained by mixing the KS local exchange $V_{x}(r)$ with a fraction $\alpha$ of the nonlocal Fock operator $V_{x}^{F}\left(r, r^{\prime}\right)=-\gamma\left(r, r^{\prime}\right) v\left(r, r^{\prime}\right)$, built using gKS orbitals in the one-particle density matrix $\gamma\left[v\left(r, r^{\prime}\right)=1 /\left|r-r^{\prime}\right|\right.$ is the Coulomb interaction]:

$$
V_{x}\left(r, r^{\prime}\right)=\alpha V_{x}^{F}\left(r, r^{\prime}\right)+(1-\alpha) V_{x}(r) .
$$

This construction is often justified from considerations based on the adiabatic-connection formula, which fix the mixing parameter to be $\alpha=0.25 .{ }^{15}$ The Coulomb interaction can then be split into a sum of a long-range and a short-range term $v\left(r, r^{\prime}\right)=v^{L R}\left(r, r^{\prime}\right)+v^{S R}\left(r, r^{\prime}\right)$, respectively, as:

$$
v\left(r, r^{\prime}\right)=\frac{\operatorname{erf}\left(\mu\left|r-r^{\prime}\right|\right)}{\left|r-r^{\prime}\right|}+\frac{1-\operatorname{erf}\left(\mu\left|r-r^{\prime}\right|\right)}{\left|r-r^{\prime}\right|} .
$$

Here both the choice of the erf error function and the parameter $\mu$ are arbitrary. Replacing the bare interaction $v$ by either term, the same separation is obtained in the local and nonlocal exchange potentials appearing in (1): $V_{x}^{F}\left(r, r^{\prime}\right)=V_{x}^{F, L R}\left(r, r^{\prime}\right)+V_{x}^{F, S R}\left(r, r^{\prime}\right)$ and $V_{x}(r)=V_{x}^{L R}(r)+$ $V_{x}^{S R}(r)$. Assuming that the effects of $V_{x}^{F, L R}\left(r, r^{\prime}\right)$ and $V_{x}^{L R}(r)$ compensate each other, the following approximation for (1) is introduced:

$$
V_{x}\left(r, r^{\prime}\right)=\alpha V_{x}^{F, S R}\left(r, r^{\prime}\right)+(1-\alpha) V_{x}^{S R}(r)+V_{x}^{L R}(r) .
$$

Regrouping the various terms contributing to the $\mathrm{xc}$ gKS potential, one finally finds:

$$
V_{\mathrm{xc}}\left(r, r^{\prime}\right)=\alpha\left[V_{x}^{F, S R}\left(r, r^{\prime}\right)-V_{x}^{S R}(r)\right]+V_{\mathrm{xc}}(r),
$$

where the correction to the KS local potential $V_{\mathrm{xc}}(r)$ (with the original Coulomb interaction) stems entirely from the first term in the right-hand side. The final approximation depends on the two parameters $\alpha$ and $\mu$. With $\mu=0, \alpha=0.25$, and using the Perdew-Burke-Ernzerhof (PBE) GGA xc potential for the local part $V_{\mathrm{xc}}(r)$, one finds the PBE0 approximation. ${ }^{15}$ In the Heyd-Scuseria-Ernzerhof (HSE06) hybrid functional, ${ }^{16}$ instead, the value of $\mu=0.2 \AA^{-1}$ is obtained by numerically fitting the results against a benchmark set of data.

More in general, both the $\alpha$ and $\mu$ parameters play the role of effective screening of the Coulomb interaction. ${ }^{19}$ The nonlocal potential (1) with $\mu=0$ can be alternatively understood as a static approximation to the many-body GW self-energy. ${ }^{11} \mathrm{By}$ identifying $1 / \alpha$ as an effective static dielectric constant $\epsilon$, $\alpha V_{x}^{F}$ can be seen as a screened exchange potential, while the local part of (1) acts as an approximation to the Coulomb hole term. ${ }^{11,20}$ In fact, varying $\alpha$ between 0 and 1 in Eq. (1), one in practice interpolates between the KS underestimation and the Hartree-Fock (HF) overestimation of band gaps, with the possibility to get close to the experimental results. Moreover, the use of a finite value for $\mu$, together with neglecting the corresponding long-range terms in Eq. (3), efficiently acts as a further screening of the Coulomb interaction. ${ }^{16,21}$ By increasing the value of $\mu$, the two-point distance $\left|r-r^{\prime}\right|$ beyond which the Coulomb interaction is cut off becomes shorter. In fact, screening the long-range Coulomb interaction is crucial for many properties in bulk materials, ${ }^{22}$ especially for small-gap and metallic systems. While for $\mu=\infty$ Eq. (3) reduces to the KS local potential $V_{\mathrm{xc}}(r)$, a finite $\mu$ tunes the correction to $\mathrm{KS}$ stemming from the difference between nonlocal Fock and local exchange terms in Eq. (3). For fixed $\alpha$, increasing $\mu$ gives less weight to this correction.

While the LDA suffers from a delocalization error, the HF instead is affected by an excess of localization. ${ }^{8}$ Hence, the inclusion in the functional (4) of a partial contribution of nonlocal Fock exchange leads to a localization correction with respect to the LDA. This affects in particular $d$ and $f$ states, which are more localized and generally suffer from a self-interaction error more than $s$ and $p$ states. ${ }^{23}$ Even though it is derived from a very different point of view, this effect of localization of $d$ and $f$ orbitals is shared also by the LDA + U approach. As we will show in the following, curing the delocalization error of the LDA is the key to get results in better qualitative agreement with experiment.

Both the LDA + U (as well as the LDA + DMFT) and the hybrid functional (4) depend on parameters (like the Hubbard $\mathrm{U}$ and $\mathrm{J}$ in the former and $\alpha$ and $\mu$ in the latter), which limits their predictivity power. However, in the hybrid functional (4) there is no need of an additional term for correcting the (unknown) spurious double counting, while this is the case for the LDA + U or LDA + DMFT. The need of a double-counting correction in fact corresponds to the introduction of a certain degree of arbitrariness in the definition of the functional. ${ }^{24}$ Moreover, while the Hubbard term in the LDA $+U$ and LDA + DMFT schemes acts only on the correlated subset of electrons, in the hybrid functional all the electrons are treated on an equal footing.

At the formal level, the KS band gap, calculated as the difference of KS eigenvalues, is not supposed to reproduce the fundamental band gap of an insulator. The difference between the two is in fact given by the derivative discontinuity of the KS xc energy. ${ }^{5-7}$ In principle, it is even possible that the exact $\mathrm{KS}$ potential yields a zero KS gap in an insulator (without necessarily implying strong correlation). In that particular case, the band gap would be equal to the derivative discontinuity. Moreover, given an approximation of the nonlocal gKS potential, it is possible to obtain a local and orbital-dependent KS potential at the same level of approximation by using an optimized effective potential (OEP) method. ${ }^{25}$ In this way, it is found that the gKS gap is always larger than the corresponding OEP-KS gap, the difference between the two being the derivative discontinuity of the orbital-dependent part of the xc energy functional. ${ }^{26}$ This underlines the role of the nonlocality of xc potential in the gKS scheme.

Modeling efficiently the static screening of the Coulomb interaction without introducing adjustable parameters would help to improve greatly the gKS scheme. At the same time, this should keep its computational cost cheaper than the more sophisticated many-body GW approximation, where the 
dynamical screening is explicitly calculated (in the randomphase approximation). In any case, being a static approximation to the many-body self-energy, the $V_{\mathrm{xc}}\left(r, r^{\prime}\right)$ functional in Eq. (4) cannot account for spectral properties related to dynamical screening effects, which for instance give rise to satellites in the photoemission spectra, ${ }^{27}$ and are instead accounted for, though in different manners, by both the GW approximation and the LDA + DMFT approach.

In the following we will use the hybrid functional form (4), as implemented in the VASP computer code..$^{28,29}$ In the case of $\mathrm{VO}_{2}$ we will discuss the effect of different choices of the two parameters $\alpha$ and $\mu$, while for the rest of the paper we will fix $\alpha=0.25$ and $\mu=0.2 \AA^{-1}$ as in the HSE06 functional. For the local $V_{\mathrm{xc}}(r)$ part we adopt a LDA xc potential, since we want to make a direct comparison with other correlated methods that treat the LDA as the uncorrelated reference. We will name this choice of the functional as the LDA-HSE06, to distinguish it from the original HSE06 functional, where the $\mathrm{PBE}$ approximation is used for the local xc potential. $\mathrm{VO}_{2}$ is the only compound among those that we have considered here that has been previously studied with the HSE06 functional. ${ }^{30}$ We will show that the LDA-HSE06 gives the same result for the density of states as the HSE06, justifying its use also for the other compounds.

\section{RESULTS AND DISCUSSION}

\section{A. $\mathrm{VO}_{2}$}

$\mathrm{VO}_{2}$ undergoes a twofold phase transition at $340 \mathrm{~K} .{ }^{31}$ The MIT is accompanied by a lowering of the symmetry of the crystal structure, from rutile to monoclinic, with a doubling of the unit cell and a dimerization of $\mathrm{V}$ atoms along the rutile $c$ axis. It has been long debated ${ }^{32,33}$ which of the two aspects, the electronic or the structural change, is the key to drive the phase transition.

In $\mathrm{VO}_{2}$ both the LDA (Ref. 34) and standard single-site DMFT (Refs. 35 and 36) are unable to get the insulating band gap, while the LDA + U (Refs. 35 and 37) has problems with the metallic phase and gives an ordered magnetic phase for the insulator, contrary to the experiment. The deficiencies of single-site DMFT have been corrected by its extension to cluster DMFT (Refs. 38 and 39), where the local impurity is taken to be a V dimer instead of a V atom, as in single-site DMFT. On the other hand, parameter-free GW calculations ${ }^{40}$ have shown that in the insulating phase, KS-LDA wave functions are not a sufficiently good approximation to quasiparticle $(\mathrm{QP})$ wave functions. The LDA error is due to an excessive delocalization of the KS wave functions at the Fermi level. They turn out to be too isotropic, underestimating the effect of the $\mathrm{V}$ dimerization along the $c$ axis, ${ }^{33}$ and the corresponding bonding-antibonding splitting of the $\mathrm{V} a_{1 g}$ states. Once this LDA error is corrected by using better QP wave functions, as obtained in a restricted self-consistent GW scheme, the results correctly reproduce the electronic properties of both phases ${ }^{40-42}$ and also show that the satellite in the photoemission spectrum of the metallic phase is related to a neutral (plasmon) excitation visible in the loss function. ${ }^{43}$

A very recent calculation ${ }^{30}$ within the HSE06 hybrid functional obtained a gap in the density of states (DOS)
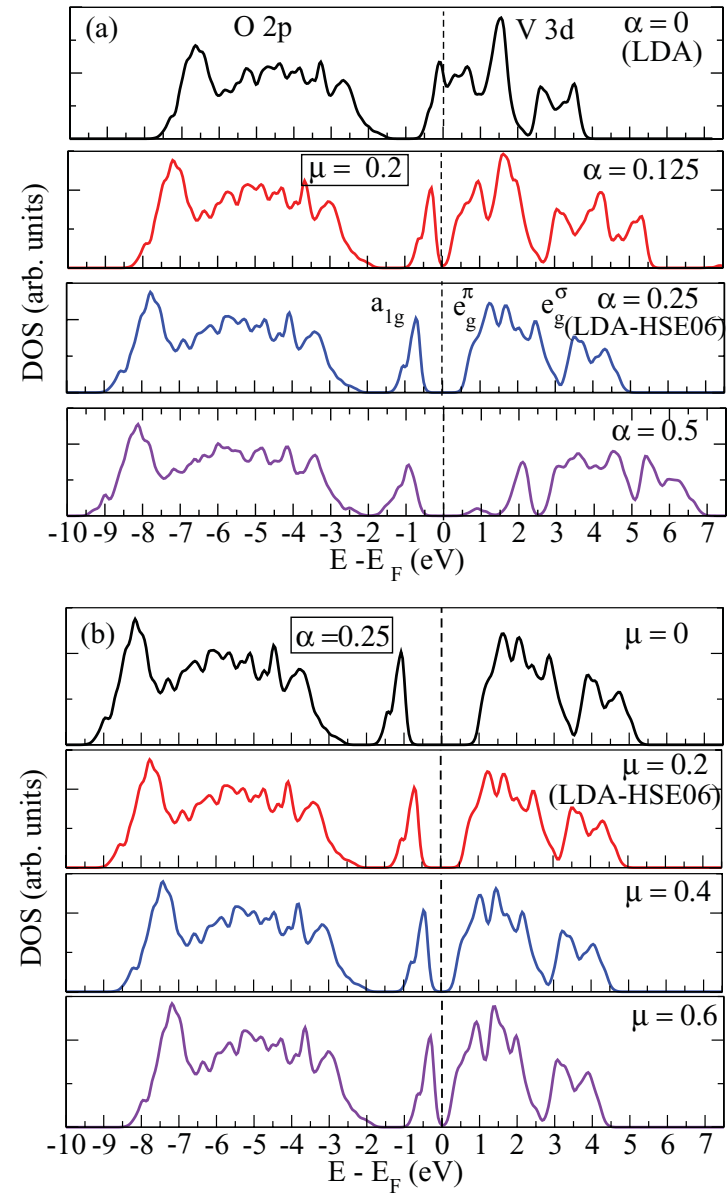

FIG. 1. (Color online) Densities of states of insulating $\mathrm{VO}_{2}$ obtained with the hybrid functional of Eq. (4) according to different choices of the mixing parameter $\alpha$ and the screening parameter $\mu$. In (a) the topmost panel corresponds to the LDA. In the other panels $\mu=0.2 \AA^{-1}$ and $\alpha$ is raised up to 0.5 . In (b) $\alpha$ is fixed to 0.25 and the results are obtained for different values of $\mu$. Here, and in all the following figures, the Fermi energy for insulators is set in the midpoint of the band gap.

of the insulator, also concluding in favor of the structural distortion as the key to explain the insulating gap. Here, more in detail, we start our investigation by analyzing the performance of the hybrid functional (4) according to different choices of the values of the mixing parameter $\alpha$ and the screening parameter $\mu$. We use the experimental crystal structures for the two phases, ${ }^{44,45}$ a $6 \times 6 \times 6 \mathrm{k}$-point grid for the insulator and a $12 \times 12 \times 12$ one for the metal. As we can see in Fig. 1, the hybrid functional (4) correctly yields a gap in the insulating phase of $\mathrm{VO}_{2}$ for many choices of the two parameters, correcting the LDA error [which is retrieved for $\alpha=0$; see topmost panel in Fig. 1(a)]. On the other hand, the DOS is highly sensitive to the values of $\alpha$ and $\mu$, both for the size of the band gap and for the separation between the highest occupied $\mathrm{V} a_{1 g}$ state and the rest of the $\mathrm{O} p$ states at higher binding energies (see Table I). Either reducing $\alpha$ [Fig. 1(a)] or increasing $\mu$ [Fig. 1(b)], the gap decreases until it disappears for $\alpha<0.125$ or $\mu>0.6 \AA^{-1}$. Keeping $\alpha$ fixed, the reduction of $\mu$ from 0.6 to $0 \AA^{-1}$ has mainly the effect of a rigid expansion of the DOS [see Fig. 1(b)]. On the contrary, 
TABLE I. Values of the fundamental band gap, the $\mathrm{O} p$ bandwidth, the $\mathrm{O} p-\mathrm{V} a_{1 g}$ separation, and the $\mathrm{V} a_{1 g}$ bandwidth in insulating $\mathrm{VO}_{2}$ depending on the different choices for the $\alpha$ and $\mu$ parameters in the hybrid functional of Eq. (4). The local $V_{\mathrm{xc}}$ is the LDA.

\begin{tabular}{lccccc}
\hline \hline$\alpha$ & $\begin{array}{c}\mu \\
\left(\AA^{-1}\right)\end{array}$ & $\begin{array}{c}\text { Band gap } \\
(\mathrm{eV})\end{array}$ & $\begin{array}{c}\mathrm{O} p \\
(\mathrm{eV})\end{array}$ & $\begin{array}{c}\mathrm{O} p-\mathrm{V} a_{1 g} \\
(\mathrm{eV})\end{array}$ & $\begin{array}{c}\mathrm{V} a_{1 g} \\
(\mathrm{eV})\end{array}$ \\
\hline 0 & & 0.00 & 6.23 & 0.96 & \\
0.125 & 0.2 & 0.00 & 6.54 & 1.02 & 0.60 \\
0.250 & 0.2 & 1.13 & 6.85 & 0.95 & 0.63 \\
0.500 & 0.2 & 1.17 & 7.58 & 0.09 & 1.16 \\
0.250 & 0.0 & 1.88 & 6.86 & 0.94 & 0.64 \\
0.250 & 0.4 & 0.65 & 6.74 & 0.96 & 0.60 \\
0.250 & 0.6 & 0.00 & 6.62 & 0.97 & 0.59 \\
\hline \hline
\end{tabular}

fixing $\mu$ and changing $\alpha$ leads also to larger modifications in the shape of all the structures appearing in the DOS, both for the occupied and the unoccupied states [see Fig. 1(a)]. Thus, the addition of nonlocal exchange to the KS-LDA functional leads to an improved qualitative agreement with experiment, i.e., a sizable band gap is obtained, without the need to invoke strong correlation effects. However, the comparison with photoemission spectra depends quantitatively on the choice that one makes for the values of the two parameters $\alpha$ and $\mu$. In fact, they both physically act as a screening of the Coulomb interaction. However, their fine tuning generally depends on an adequate microscopic description of the screening in the actual material.

We have also calculated the equilibrium volumes $V$ of the insulating phase of $\mathrm{VO}_{2}$ according to different parametrizations of the hybrid functional (see Table II). All the results are very close to the experimental value $V_{\exp }$, with a discrepancy of 5\% at most. As generally found in the literature, also for $\mathrm{VO}_{2}$ the LDA overbinds and the PBE underbinds. In both cases, the difference with respect to experiment is only $3 \%$, confirming previous results. ${ }^{33,40}$ DFT is an exact theory for the ground state and the LDA and PBE are, even for $\mathrm{VO}_{2}$, already good approximations. The effect of the nonlocal exchange is a general reduction of the LDA and PBE volumes, resulting in a slight underestimation of the experimental value. We find that the choice of both the approximation for the local $V_{\mathrm{xc}}$ (either the LDA or PBE) and the values of the $\alpha$ and $\mu$

TABLE II. Equilibrium volumes $V$ of the insulating $\mathrm{VO}_{2}$ divided by the experimental volume $V_{\text {exp }}$, calculated with different choices of the $\alpha$ and $\mu$ parameters and the approximation for the local $V_{\mathrm{xc}}$. The first row corresponds to the local LDA and PBE approximations and the third row to the LDA-HSE06 and HSE06 parametrizations.

\begin{tabular}{lccc}
\hline \hline$\alpha$ & $\mu$ & $\begin{array}{c}\text { Local } V_{\text {xc }} \text { LDA } \\
V / V_{\text {exp }}\end{array}$ & $\begin{array}{c}\text { Local } V_{\text {xc }} \text { PBE } \\
V / V_{\text {exp }}\end{array}$ \\
\hline 0 & & 0.97 & 1.03 \\
0.125 & 0.2 & 0.96 & 1.00 \\
0.250 & 0.2 & 0.95 & 0.98 \\
0.500 & 0.2 & 0.95 & 0.95 \\
0.250 & 0.0 & 0.95 & 0.98 \\
0.250 & 0.4 & 0.95 & 0.98 \\
0.250 & 0.6 & 0.95 & 0.99 \\
\hline \hline
\end{tabular}
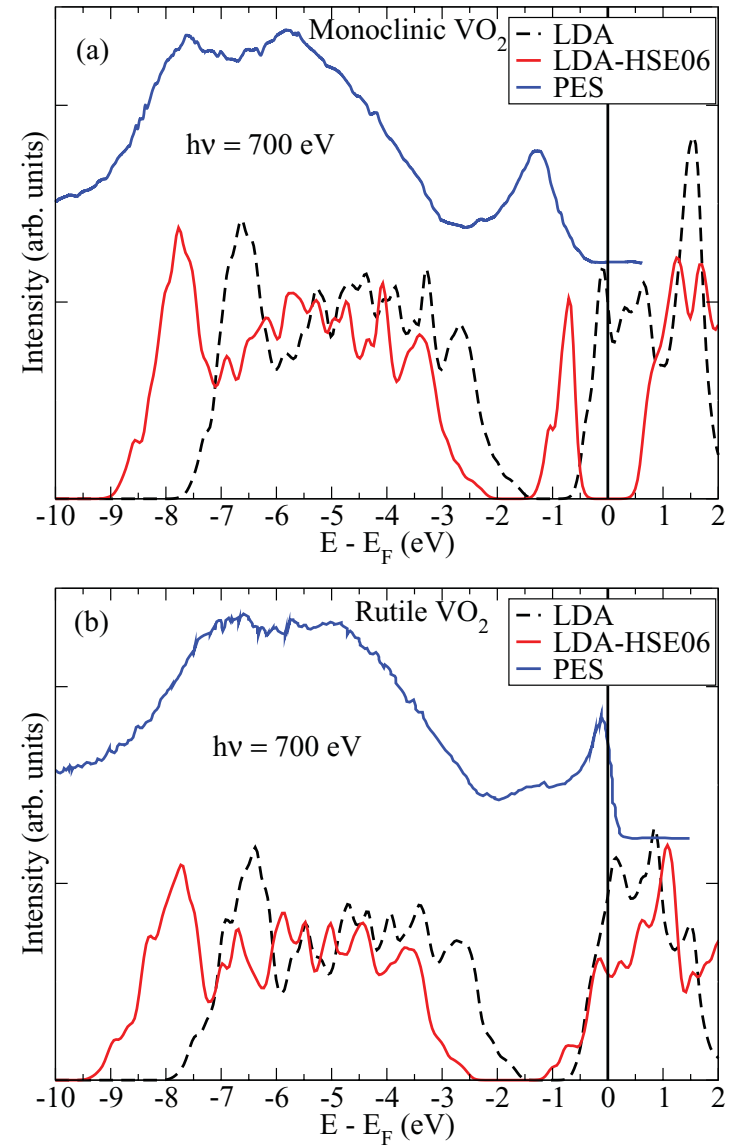

FIG. 2. (Color online) Comparison of the calculated density of states of (upper panel) insulating monoclinic and (bottom panel) metallic rutile phases of $\mathrm{VO}_{2}$ with the experimental photoemission spectra from Ref. 46. The MIT is correctly reproduced by the LDA-HSE06 hybrid functional, while the LDA DOS is always metallic.

parameters affect only to a small extent the results obtained with hybrid functionals. In particular, the difference between HSE06 and LDA-HSE06 results is smaller than between their local counterparts, the PBE and LDA, respectively.

In Fig. 2 we compare the calculated DOS for both phases of $\mathrm{VO}_{2}$ with the experimental photoemission spectra. ${ }^{46}$ When comparing with experiments, it is essential to have bulksensitive photoemission data, which can be obtained by using high-energy photons as in an x-ray photoemission (XPS) or, even better, a hard $\mathrm{x}$-ray photoemission (HAXPES) setup. ${ }^{47}$ In fact, at low photon energies, photoemission spectroscopy is mainly surface sensitive. However, in these materials the electronic properties of the surfaces are generally different from the bulk. Here, and in the rest of paper, we use $\mu=0.2 \AA^{-1}$ and $\alpha=0.25$, in agreement with the HSE06 parametrization. With this choice we find a gap of $1.13 \mathrm{eV}$, in excellent agreement with the value of $1.1 \mathrm{eV}$ from Ref. 30, where a GGA instead of a KS-LDA local functional has been used though. This shows that using either a LDA or GGA KS functional does not have an influence on the DOS. Both results overestimate the experimental band gap, which is $0.6 \mathrm{eV}$ in the insulator. ${ }^{46}$ Nevertheless, the HSE06 hybrid functional is able 
to describe correctly the MIT, contrary to the LDA, LDA + U, and LDA + DMFT.

\section{B. $\mathrm{V}_{2} \mathrm{O}_{3}$}

Like $\mathrm{VO}_{2}, \mathrm{~V}_{2} \mathrm{O}_{3}$ is a time-honored correlated material, whose great interest is due to its temperature-induced MIT. ${ }^{18,48}$ At $T>154 \mathrm{~K}$ it is a paramagnetic metal, while at low temperature it becomes an antiferromagnetic insulator and undergoes a crystal distortion from a corundum to a monoclinic structure. The phase diagram is made more complex by doping with $\mathrm{Cr}$, which induces a different isostructural MIT to a paramagnetic phase. The antiferromagnetic phase has been studied in the LDA + U (Ref. 49) (also followed by a perturbative GW calculation ${ }^{50}$ ) and LDA + DMFT (Ref. 51), while the spectral properties of the metallic phase have been extensively analyzed in the LDA + DMFT (Refs. 51-54) and in the GW (Ref. 55).

Here we take the experimental lattice parameters of the pure compounds. ${ }^{56,57}$ We consider different magnetic configurations for the insulating phase, in order to analyze their influence on the electronic structure and compare hybrid functional results with those found in the $\mathrm{LDA}+\mathrm{U}^{49}$ In the calculation we used a $6 \times 6 \times 6$ grid of $\mathbf{k}$ points for the insulator, which becomes $10 \times 10 \times 10$ for the metal. In the experimental magnetic structure (AFI1), ${ }^{58}$ each $\mathrm{V}$ atom has one spin-parallel neighbor and two spin-antiparallel neighbors in the (distorted) hexagonal planes of the monoclinic crystal, while the coupling between neighbors in different planes is ferromagnetic [see inset to Fig. 3(a)]. We also considered a ferromagnetic (FM) order and an alternative antiferromagnetic (AFI2) order, in which, with respect to the experiment, the interplane magnetic coupling is inverted, while it is unchanged inside the planes [see inset to Fig. 3(b)]. The LDA always yields a metal, regardless of the magnetic structures. Instead, the LDA-HSE06 gives an insulator for all the magnetic configurations considered. The band gap is 1.80 $\mathrm{eV}$ in the AFI1, much larger than the $0.66 \mathrm{eV}$ experimental optical gap. ${ }^{59}$ The two antiferromagnetic DOSs turn out to be very similar, while for the FM the DOS [see Fig. 3(c)] is quite different and the band gap reduces to $0.7 \mathrm{eV}$. In the LDA + U calculation, ${ }^{49}$ the FM DOS is half metallic, and the experimental antiferromagnetic structure has a $0.7 \mathrm{eV}$ gap. The LDA-HSE06 consistently overestimates the band gap in all the magnetic structures. This result is connected to an overestimation of the local magnetic moment, which in the LDA-HSE06 is $1.8 \mu_{B}$ for the AFI structures and $1.9 \mu_{B}$ for the FM, whereas experimentally it is $1.2 \mu_{B} /(\mathrm{V}$ at.). Similarly to the $\mathrm{LDA}+\mathrm{U}$, the ground-state total energy difference between different magnetic structures is rather small, with the experimental AFI1 structure being the one with lowest total energy.

In Fig. 4 we compare the calculated DOSs with the experimental HAXPES results from Ref. 60. As in $\mathrm{VO}_{2}$ (see Sec. III A), the LDA-HSE06 is able to reproduce the MIT, contrary to the LDA. At the same time, it also corrects the LDA underestimation of the binding energy of the $\mathrm{O}$ $p$ states in both phases. This is a clear illustration of the advantage of treating all the electrons on equal footing (in the LDA + U or LDA + DMFT, instead, the position of $\mathrm{O} p$
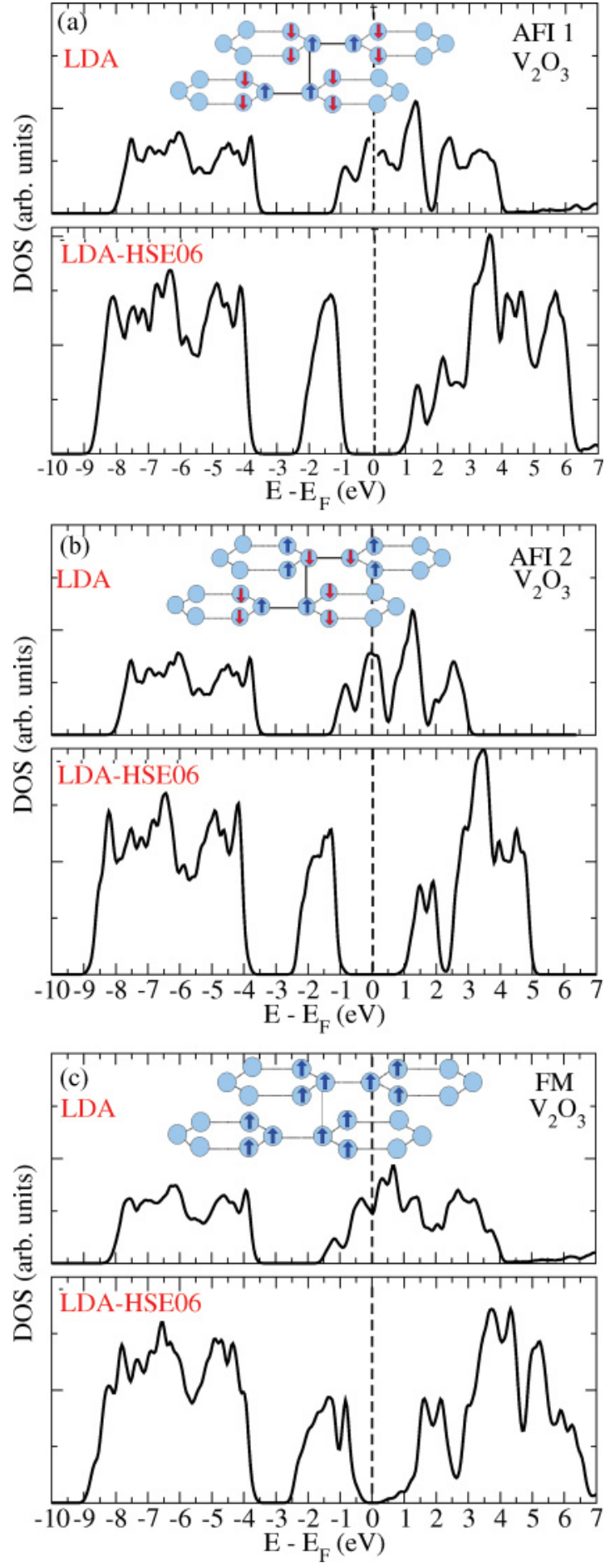

FIG. 3. (Color online) Density of states of monoclinic insulating $\mathrm{V}_{2} \mathrm{O}_{3}$, calculated both in the LDA and LDA-HSE06, according to the different considered magnetic structures. The magnetic order is visualized in the insets to each panel, where the light blue circles schematically represent $\mathrm{V}$ atoms in the (distorted) hexagonal planes that characterize the monoclinic crystal structure and the arrows display the directions of the local magnetic moments.

states is essentially the same as in the LDA). However, the LDA-HSE06 overestimates the bandwidth of the top valence $a_{1 g}$ states, and the band gap in the insulator. 

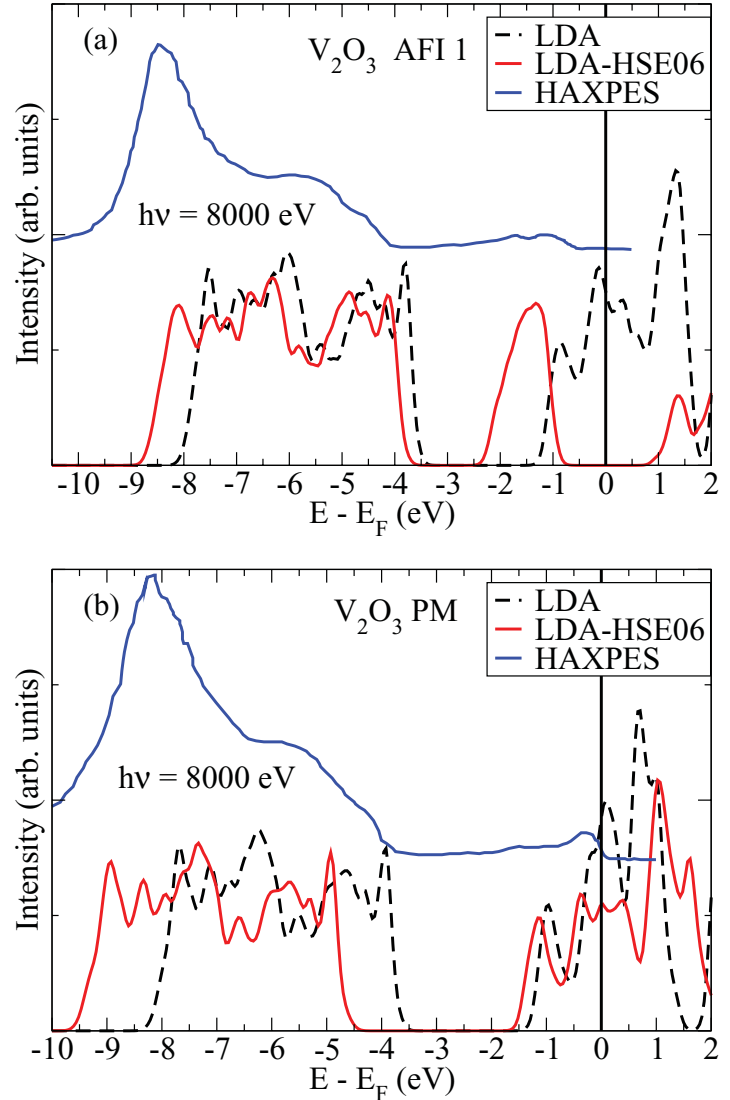

FIG. 4. (Color online) Comparison between the DOSs calculated in the LDA and LDA-HSE06 and the experimental photoemission spectra from Ref. 60. (a) Antiferromagnetic insulating phase and (b) paramagnetic metallic phases of $\mathrm{V}_{2} \mathrm{O}_{3}$.

\section{C. $\mathrm{Ti}_{2} \mathrm{O}_{3}$}

$\mathrm{Ti}_{2} \mathrm{O}_{3}$ has a corundum crystal structure like metallic $\mathrm{V}_{2} \mathrm{O}_{3}$ and a formal $d^{1}$ configuration like $\mathrm{VO}_{2}$. Below $400 \mathrm{~K}$ it is insulating and above $600 \mathrm{~K}$ it is metallic, undergoing a very broad temperature-induced MIT (Refs. 31 and 61). Moreover, neither phase is magnetically ordered, ${ }^{62}$ and, contrary to both $\mathrm{VO}_{2}$ and $\mathrm{V}_{2} \mathrm{O}_{3}$, the MIT in $\mathrm{Ti}_{2} \mathrm{O}_{3}$ is isostructural. ${ }^{63}$ In fact, by raising the temperature only an increase of the $c / a$ ratio is observed, accompanied by an increase of the Ti dimer distance along the $c$ axis. Here we consider two crystal structures with lattice parameters measured at 296 and 868 K (Ref. 63), where $\mathrm{Ti}_{2} \mathrm{O}_{3}$ is insulating and metallic, respectively. In our calculations we used a $6 \times 6 \times 6 \mathbf{k}$-point grid for the insulating phase and a $8 \times 8 \times 8$ one for the metal.

The presence of $\mathrm{Ti}$ dimers along the $c$ axis leads to a large bonding-antibonding splitting of the $a_{1 g}$ states, as in the insulating $\mathrm{VO}_{2}$ case. Here, without additional structural changes, we can directly relate the shortening of the Ti dimer distance with the increase of the $a_{1 g}$ bonding-antibonding splitting, and hence the band-gap opening between the bonding $a_{1 g}$ and the $e_{g}^{\pi}$ states in the $t_{2 g}$ subband of Ti $3 d$ orbitals. Also in the present case, this effect is masked in the LDA by its underestimation of the anisotropy introduced by the Ti dimers. For this reason, the LDA DOS remains metallic also at low temperatures. ${ }^{64,65}$ The relevance of the Ti dimers is underlined also by the fact that, as in $\mathrm{VO}_{2}$, single-site DMFT is not able
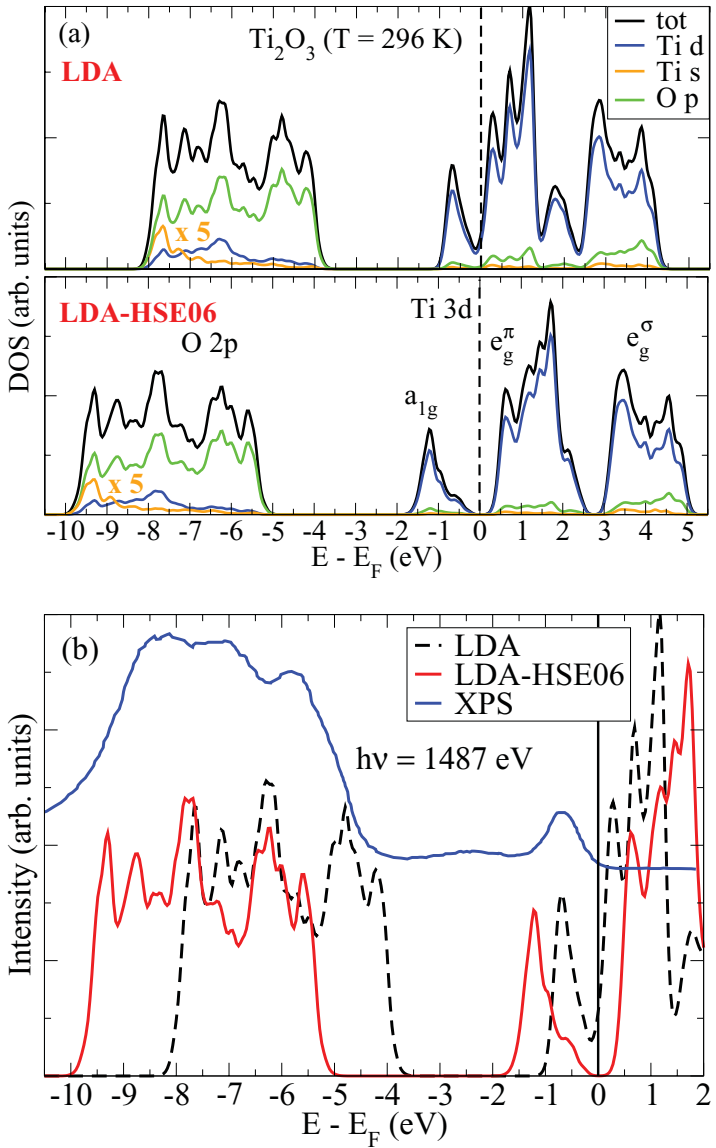

FIG. 5. (Color online) (a) LDA and LDA-HSE06 densities of states (upper and bottom panels, respectively), together with the DOSs projected onto Ti $s$, Ti $d$, and $\mathrm{O} p$ states, for insulating $\mathrm{Ti}_{2} \mathrm{O}_{3}$ at room temperature. (b) Comparison of the total DOS in the LDA and HSE06 with the XPS spectrum from Ref. 67.

to obtain the insulating phase, which is instead reproduced by a cluster DMFT calculation ${ }^{66}$ for which the local impurity is given by the pair of $\mathrm{Ti}$ atoms.

The effect of the LDA-HSE06 corrections over the LDA [see Figs. 5(a) and 6] is visible for both phases: (i) at the Fermi energy, where the top valence band is split off from the bottom of the conduction states, leading to the gap opening in the insulator and to a strong reduction of the spectral weight at the Fermi energy in the metal; (ii) in the transfer of spectral weight in the unoccupied $\mathrm{Ti} t_{2 g}$ band toward the high-energy part of the band; (iii) in the increasing of the separation between $\mathrm{Ti}$ $d$ states and $\mathrm{O} p$ states, as a consequence of a rigid shift of the latter. In general, LDA-HSE06 results do not change the hybridization character of the bands with respect to the LDA.

At room temperature the LDA-HSE06 gives a $0.57 \mathrm{eV}$ gap, which is much larger than the $0.11 \mathrm{eV}$ estimate from conductivity and thermoelectric coefficient measurements. ${ }^{68}$ However, contrary to $\mathrm{HF}$ calculations, ${ }^{69}$ the spin-polarized LDA-HSE06 correctly yields a nonmagnetic solution also in the insulating phase. This result is important since it shows that within the hybrid functional the existence of a gap is not necessarily linked to the presence of magnetic order, as it often happens in the LDA + U. Moreover, the LDA-HSE06 correctly describes also the metallic phase and the MIT (see 


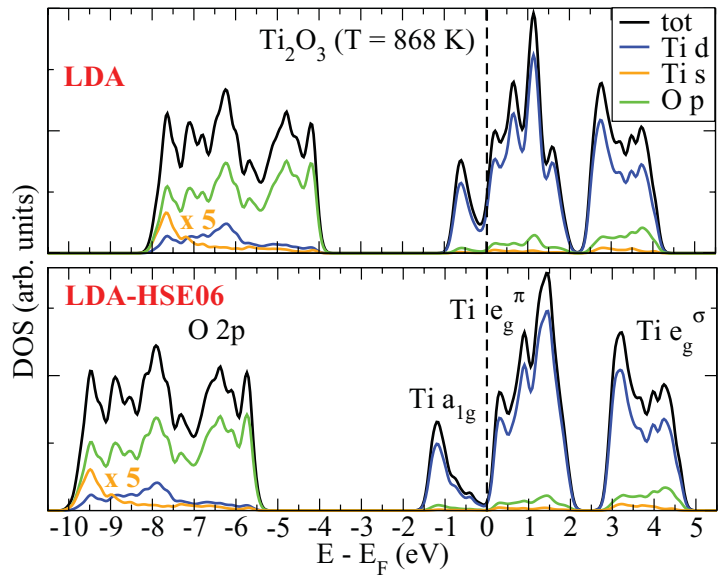

FIG. 6. (Color online) Comparison between the LDA and LDAHSE06 DOSs and Ti $s$, Ti $d$, and O $p$ projected DOSs, calculated for metallic $\mathrm{Ti}_{2} \mathrm{O}_{3}$ at $T=868 \mathrm{~K}$.

Fig. 6), which is often problematic within the LDA + U. Thus, these results appear to have two advantages of the HSE06 with respect to the LDA + U in nonmagnetic insulators and metals.

In the XPS spectrum [at $h v=1487 \mathrm{eV}$, reproduced in Fig. $5(\mathrm{~b})]^{67}$ for the insulating phase at room temperature, a satellite is clearly visible at $2.4 \mathrm{eV}$, between the Ti $3 d$ peak at $0.7 \mathrm{eV}$ and the broad $\mathrm{O} 2 p$ band at 4 to $10 \mathrm{eV}$. This satellite cannot be obtained with the static hybrid functional employed here, but is absent also in the cluster DMFT calculation. ${ }^{66}$ The HAXPES spectrum $(h v=5931 \mathrm{eV}),{ }^{67}$ which is dominated by the cation $s$ contribution as in the vanadium oxides ${ }^{43,55}$ (see Fig. 4), confirms that the satellite present in the XPS spectrum is a genuine bulk feature of insulating $\mathrm{Ti}_{2} \mathrm{O}_{3}$ (instead, experimental photoemission results for the metallic phase are not available). Overall, the LDA-HSE06 results [see Fig. 5(b)] compare much better with the experimental spectra than the LDA.

\section{D. $\mathrm{LaTiO}_{3}$ and $\mathrm{YTiO}_{3}$}

In a seminal paper, Fujimori et al. ${ }^{70}$ considered a series of $d^{1}$ transition metal oxides. On the basis of a single-band Hubbard model, they explained the opening of the band gap, going from metallic $\mathrm{VO}_{2}$ and $\mathrm{SrVO}_{3}$ to insulating $\mathrm{YTiO}_{3}$ and $\mathrm{LaTiO}_{3}$, as the progressive increase of the ratio between the Hubbard $\mathrm{U}$ and the $d$ bandwidth, which is accompanied by the transfer of spectral weight from the quasiparticle peak at the Fermi energy in the metals to the Hubbard bands in the insulators. Within this view, both $\mathrm{LaTiO}_{3}$ and $\mathrm{YTiO}_{3}$ are Mott insulators, with a gap opening between the lower and the upper Hubbard bands. Thus a band-structure description would not be able to reproduce these incoherent atomiclike excitations in the spectra, and hence the gap. In fact, KS-LDA band structures are metallic for both compounds, ${ }^{71,72}$ while LDA $+\mathrm{U}^{71-73}$ and LDA + DMFT ${ }^{74-76}$ calculations correctly yield a gap.

At low temperatures both compounds order magnetically. Below $148 \mathrm{~K} \mathrm{LaTiO}_{3}$ displays a G-type antiferromagnetic order with a local magnetic moment of $0.57 \mu_{B}$ (Ref. 77). $\mathrm{YTiO}_{3}$ is ferromagnetic below $29 \mathrm{~K}$, where the magnetic moment is $0.8 \mu_{B} /$ (Ti at.) (Ref. 78). It has been much debated whether the magnetic properties of these compounds can be
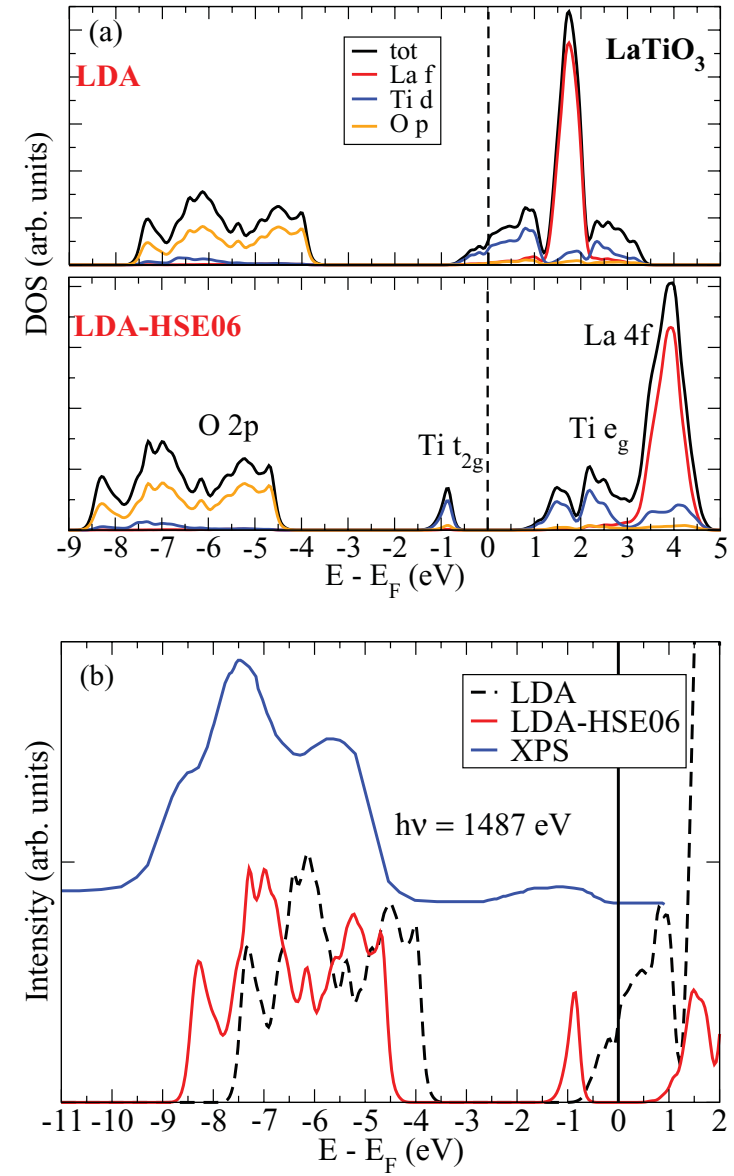

FIG. 7. (Color online) (a) DOS and projected DOS for $\mathrm{LaTiO}_{3}$ calculated in the LDA and LDA-HSE06 and (b) comparison with experimental photoemission spectra from Ref. 83 .

explained in terms of the formation of an orbital liquid or orbital ordering. ${ }^{79-81}$

Here we use the low-temperature experimental crystal structures from Refs. 77 and 82 , and a $4 \times 4 \times 4$ grid of k points. While $\mathrm{LaTiO}_{3}$ is nonmagnetic in the LDA, in the LDA-HSE06 the local magnetic moment is $0.76 \mu_{B}$, overestimating the experimental value of $0.57 \mu_{B}$. Also in ferromagnetic $\mathrm{YTiO}_{3}$, in the LDA-HSE06 it increases up to $0.84 \mu_{B}$ from $0.7 \mu_{B}$ in the LDA, reaching a similar value as in the GGA $+\mathrm{U}^{73}$

Also in these compounds, the LDA-HSE06 gives insulating densities of states [see Figs. 7(a) and 8(a) for $\mathrm{LaTiO}_{3}$ and $\mathrm{YTiO}_{3}$, respectively]. This is the result of the splitting off of a Ti $t_{2 g}$ band from the states crossing the Fermi level in the LDA. In the case of $\mathrm{YTiO}_{3}$ only spin-up states contribute to the occupied $t_{2 g}$ band. Thus, these results for both compounds seem to be in contrast with the traditional interpretation of the topmost occupied state as an incoherent lower Hubbard band. ${ }^{70}$ In fact, the peak in the experimental spectra ${ }^{83}$ [see Figs. 7(b) and $8(b)]$ is matched, at least partially, by this (coherent) Ti $t_{2 g}$ band. Moreover, within the hybrid functional scheme, the opening of the gap with respect to the metallic LDA DOS is due to nonlocal exchange. However, contrary to the experiment, in the LDA-HSE06 the bandwidth of these valence Ti $t_{2 g}$ states is larger in $\mathrm{YTiO}_{3}$ than in $\mathrm{LaTiO}_{3}$. At the same time, also 

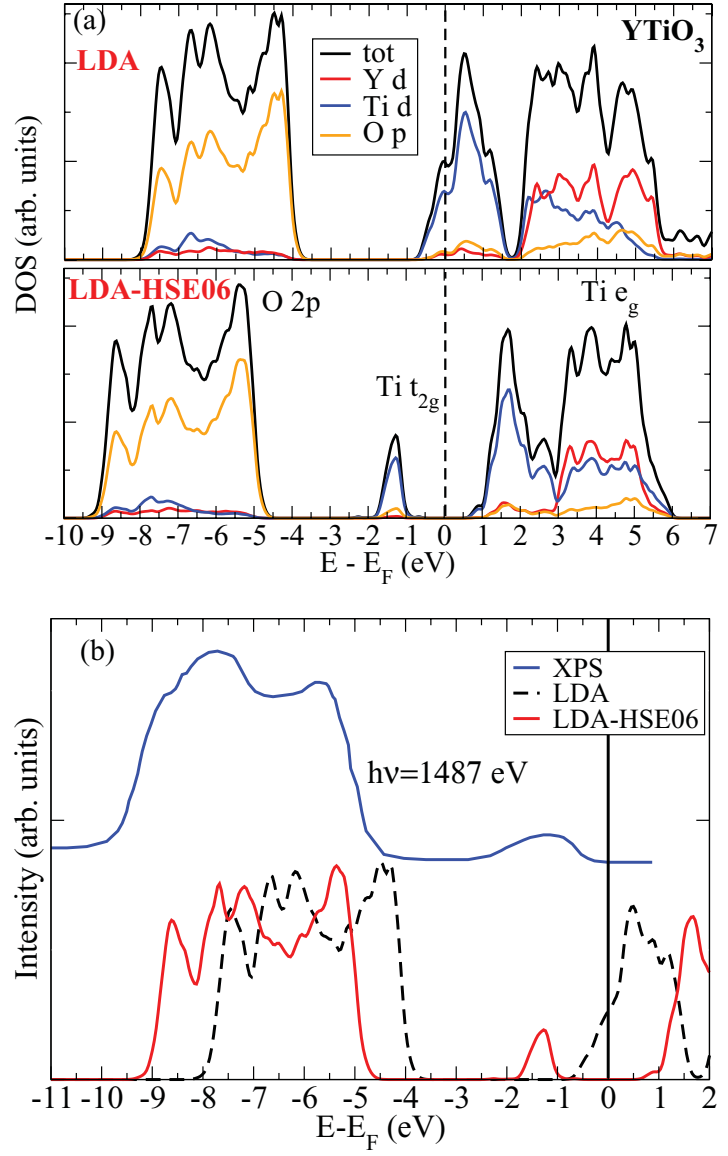

FIG. 8. (Color online) (a) DOS and projected DOS for $\mathrm{YTiO}_{3}$ calculated in the LDA and LDA-HSE06 and (b) comparison with experimental photoemission spectra from Ref. 83.

the band gap is larger in $\mathrm{LaTiO}_{3}(1.74 \mathrm{eV})$ than in $\mathrm{YTiO}_{3}$ $(1.41 \mathrm{eV})$, while the experimental optical gap is $0.2 \mathrm{eV}$ for $\mathrm{LaTiO}_{3}$ (Ref. 84) and $0.7 \mathrm{eV}$ for $\mathrm{YTiO}_{3}$ (Ref. 85). In both compounds the band gap is overestimated, while the occupied $\mathrm{Ti} t_{2 g}$ bandwidth is underestimated, also for possible dynamical effects $^{74}$ that are missing here.

While, as in all the other compounds, the hybridization between $\mathrm{O} p$ states and Ti $d$ states does not change much between the LDA and LDA-HSE06 [see Figs. 7(a) and 8(a)], a larger effect is seen here for the unoccupied La $f$ states in $\mathrm{LaTiO}_{3}$. In the LDA they are located in the middle of the conduction band, while in the LDA-HSE06 they are shifted to the upper end of the band. The band gap opening between Ti $d$ states and the upshift of the La $f$ states can been obtained in the GGA + U approach ${ }^{73}$ only with the simultaneous use of two (different) Hubbard $\mathrm{U}$ values applied to Ti $d$ and La $f$ states. Within hybrid functionals this result emerges naturally as a consequence of the localization of these states (the nonlocal exchange corrects the LDA delocalization error treating all the electrons on equal footing).

Both $\mathrm{LaTiO}_{3}$ and $\mathrm{YTiO}_{3}$ remain insulating also above the corresponding (Néel or Curie) temperatures where they loose their magnetic order. A spin-unpolarized HSE06 calculation would not be able to obtain a gap in this case. In agreement with the Mott picture, ${ }^{86}$ while the long-range magnetic order is not essential to have an insulator, the electronic spins do matter. In fact, $\mathrm{LaTiO}_{3}$ and $\mathrm{YTiO}_{3}$ in the disordered phases are both paramagnetic. Similarly, above the Néel temperatures transition-metal monoxides are also paramagnetic insulators. For these compounds it was recently shown that a calculation, based on the self-interaction-corrected (SIC) functional and taking explicitly into account the disordered local moments of the paramagnetic phase, was able to correctly describe the insulating phases. ${ }^{87}$ Thus, a similar calculation would be suitable in the (disordered) paramagnetic phase also for the present perovskite compounds (for which a modified SIC implementation has been recently used for the magnetically ordered phases). ${ }^{88}$

\section{CONCLUSIONS}

The hybrid functionals employed in the present work are not explicitly designed to treat electronic correlations. Nevertheless, we have shown that the inclusion of nonlocal Fock exchange is essential to cure the fundamental problem of getting metallic band structures in the Kohn-Sham LDA in the insulating phases of several correlated transition-metal oxides, as those that have been discussed here: $\mathrm{VO}_{2}, \mathrm{~V}_{2} \mathrm{O}_{3}, \mathrm{Ti}_{2} \mathrm{O}_{3}$, $\mathrm{LaTiO}_{3}$, and $\mathrm{YTiO}_{3}$. Analogous results have been obtained for instance also by Rödl et al. ${ }^{17}$ in the series of transition-metal monoxides, where hybrid functionals were also used as an improved starting point for one-shot GW calculations. ${ }^{89}$

Thus, a common conclusion emerges from the study of all these correlated transition-metal oxides using the LDA-HSE06 (see Table III): (i) With respect to the LDA, besides providing a finite band gap, the LDA-HSE06 also correct the position of the $\mathrm{O} p$ states. This is a clear advantage with respect to other approaches (the LDA + U, LDA + DMFT, etc.) stemming from treating all the electrons on the same footing. (ii) With respect to the LDA + U another advantage is the consistent treatment of insulators and metals and the fact that hybrid functionals do not yield an insulator together with magnetic long-range order. (iii) The LDA-HSE06 parametrization overestimates the band gap in all the compounds considered here, and, in general, the results are sensitive to the choice of the parameters (as in the LDA + U and LDA + DMFT) used to build the functional. (iv) Hybrid functionals miss completely dynamical screening

TABLE III. Summary of the results for the local magnetic moment for the magnetically ordered compounds and for the band gaps of the insulating phases.

\begin{tabular}{lccc}
\hline \hline & \multicolumn{3}{c}{ Local magnetic moment $\left(\mu_{B}\right)$} \\
\cline { 2 - 4 } & Expt. & LDA & LDA-HSE06 \\
\hline $\mathrm{V}_{2} \mathrm{O}_{3}$ & 1.2 & 1.22 & 1.8 \\
$\mathrm{LaTiO}_{3}$ & 0.57 & 0 & 0.76 \\
$\mathrm{YTiO}_{3}$ & 0.8 & 0.7 & 0.84 \\
& & Band gap $(\mathrm{eV})$ & \\
$\mathrm{VO}_{2}$ & Expt. & LDA & LDA-HSE06 \\
$\mathrm{V}_{2} \mathrm{O}_{3}$ & 0.6 & 0 & 1.13 \\
$\mathrm{Ti}_{2} \mathrm{O}_{3}$ & 0.66 & 0 & 1.80 \\
$\mathrm{LaTiO}_{3}$ & 0.11 & 0 & 0.57 \\
$\mathrm{YTiO}_{3}$ & 0.2 & 0 & 1.74 \\
\hline \hline
\end{tabular}


effects, which are essential for the description of satellites in photoemission spectra. ${ }^{27}$

Therefore, it is evident that hybrid functionals cannot be (and they are not meant to be) the final answer for the description of spectral properties of correlated transition-metal oxides. However, also in these compounds, hybrid functionals demonstratebeing very useful for the discussion of the role of nonlocal exchange, upon which a clean analysis of the effects of (dynamical) electronic correlation can be then established.

\section{ACKNOWLEDGMENTS}

We thank Lucia Reining for many fruitful discussions. Financial support was provided by Spanish MEC (Grants No. FIS2011-65702-C02-01 and No. PIB2010US-00652), ACI-Promociona (Grant No. ACI2009-1036), Grupos Consolidados UPV/EHU del Gobierno Vasco (Grants No. IT-319-07), and the European Research Council Advanced Grant DYNamo (ERC-2010-AdG -Proposal No. 267374). Computational time was granted by i2basque and BSC "Red Española de Supercomputación."
${ }^{1}$ W. Kohn and L. J. Sham, Phys. Rev. A 140, 1133 (1965).

${ }^{2}$ J. P. Perdew, K. Burke, and M. Ernzerhof, Phys. Rev. Lett. 77, 3865 (1996); 78, 1396(E) (1997).

${ }^{3}$ V. I. Anisimov, J. Zaanen, and O. K. Andersen, Phys. Rev. B 44, 943 (1991).

${ }^{4}$ A. Georges, G. Kotliar, W. Krauth, and M. J. Rozenberg, Rev. Mod. Phys. 68, 13 (1996).

${ }^{5}$ L. J. Sham and M. Schlüter, Phys. Rev. Lett. 51, 1888 (1983).

${ }^{6}$ J. P. Perdew and M. Levy, Phys. Rev. Lett. 51, 1884 (1983).

${ }^{7}$ R. W. Godby, M. Schlüter, and L. J. Sham, Phys. Rev. Lett. 56, 2415 (1986).

${ }^{8}$ P. Mori-Sánchez, A. J. Cohen, and W. T. Yang, Phys. Rev. Lett. 100, 146401 (2008).

${ }^{9}$ G. Onida, L. Reining, and A. Rubio, Rev. Mod. Phys. 74, 601 (2002).

${ }^{10}$ M. van Schilfgaarde, T. Kotani, and S. Faleev, Phys. Rev. Lett. 96, 226402 (2006).

${ }^{11}$ L. Hedin, Phys. Rev. A 139, 796 (1965).

${ }^{12}$ M. S. Hybertsen and S. G. Louie, Phys. Rev. B 34, 5390 (1986).

${ }^{13}$ A. Seidl, A. Görling, P. Vogl, J. A. Majewski, and M. Levy, Phys. Rev. B 53, 3764 (1996).

${ }^{14}$ D. M. Bylander and L. Kleinman, Phys. Rev. B 41, 7868 (1990).

${ }^{15}$ J. P. Perdew, M. Ernzerhof, and K. Burke, J. Chem. Phys. 105, 9982 (1996); C. Adamo and V. Barone, ibid. 110, 6158 (1999).

${ }^{16}$ J. Heyd, G. E. Scuseria, and M. Ernzerhof, J. Chem. Phys. 118, 8207 (2003); 124, 219906(E) (2006).

${ }^{17}$ C. Rödl, F. Fuchs, J. Furthmüller, and F. Bechstedt, Phys. Rev. B 79, 235114 (2009).

${ }^{18}$ M. Imada, A. Fujimori, and Y. Tokura, Rev. Mod. Phys. 70, 1039 (1998).

${ }^{19}$ M. A. L. Marques, J. Vidal, M. J. T. Oliveira, L. Reining, and S. Botti, Phys. Rev. B 83, 035119 (2011).

${ }^{20}$ F. Gygi and A. Baldereschi, Phys. Rev. Lett. 62, 2160 (1989).

${ }^{21}$ A. V. Krukau, O. A. Vydrov, A. F. Izmaylov, and G. E. Scuseria, J. Chem. Phys. 125, 224106 (2006).

${ }^{22}$ See, e.g., F. Bruneval, F. Sottile, V. Olevano, and L. Reining, J. Chem. Phys. 124, 144113 (2006).

${ }^{23}$ P. Mori-Sánchez, A. J. Cohen, and W. T. Yang, J. Chem. Phys. 125, 201102 (2006).

${ }^{24}$ E. R. Ylvisaker, W. E. Pickett, and K. Koepernik, Phys. Rev. B 79, 035103 (2009).

${ }^{25}$ R. T. Sharp and G. K. Horton, Phys. Rev. 90, 317 (1953); J. D. Talman and W. F. Shadwick, Phys. Rev. A 14, 36 (1976).

${ }^{26}$ M. Grüning, A. Marini, and A. Rubio, Phys. Rev. B 74, 161103R (2006).
${ }^{27}$ M. Guzzo, G. Lani, F. Sottile, P. Romaniello, M. Gatti, J. J. Kas, J. J. Rehr, M. G. Silly, F. Sirotti, and L. Reining, Phys. Rev. Lett. 107, 166401 (2011).

${ }^{28}$ G. Kresse and J. Furthmüller, Comput. Mater. Sci. 6, 15 (1996); Phys. Rev. B 54, 11169 (1996).

${ }^{29}$ J. Paier, M. Marsman, K. Hummer, G. Kresse, I. C. Gerber, and J. G. Ángyán, J. Chem. Phys. 124, 154709 (2006); 125, 249901(E) (2006).

${ }^{30}$ V. Eyert, Phys. Rev. Lett. 107, 016401 (2011).

${ }^{31}$ F. J. Morin, Phys. Rev. Lett. 3, 34 (1959).

${ }^{32}$ A. Zylbersztejn and N. F. Mott, Phys. Rev. B 11, 4383 (1975).

${ }^{33}$ R. M. Wentzcovitch, W. W. Schulz, and P. B. Allen, Phys. Rev. Lett. 72, 3389 (1994).

${ }^{34}$ V. Eyert, Ann. Phys. (Berlin) 11, 650 (2002).

${ }^{35}$ A. Liebsch, H. Ishida, and G. Bihlmayer, Phys. Rev. B 71, 085109 (2005).

${ }^{36}$ M. S. Laad, L. Craco, and E. Müller-Hartmann, Phys. Rev. B 73, 195120 (2006).

${ }^{37}$ A. V. Kozhevnikov, V. I. Anisimov, and M. A. Korotin, Phys. Met. Metall. 104, 215 (2007).

${ }^{38}$ S. Biermann, A. Poteryaev, A. I. Lichtenstein, and A. Georges, Phys. Rev. Lett. 94, 026404 (2005).

${ }^{39}$ B. Lazarovits, K. Kim, K. Haule, and G. Kotliar, Phys. Rev. B 81, 115117 (2010).

${ }^{40}$ M. Gatti, F. Bruneval, V. Olevano, and L. Reining, Phys. Rev. Lett. 99, 266402 (2007)

${ }^{41}$ A. Continenza, S. Massidda, and M. Posternak, Phys. Rev. B 60, 15 (1999).

${ }^{42}$ R. Sakuma, T. Miyake, and F. Aryasetiawan, Phys. Rev. B 78, 075106 (2008).

${ }^{43}$ M. Gatti, G. Panaccione, and L. Reining (unpublished).

${ }^{44}$ D. B. McWhan, M. Marezio, J. P. Remeika, and P. D. Dernier, Phys. Rev. B 10, 490 (1974).

${ }^{45}$ J. M. Longo and P. Kierkegaard, Acta Chem. Scand. 24, 420 (1970).

${ }^{46}$ T. C. Koethe, Z. Hu, M. W. Haverkort, C. Schüßler-Langeheine, F. Venturini, N. B. Brookes, O. Tjernberg, W. Reichelt, H. H. Hsieh, H.-J. Lin, C. T. Chen, and L. H. Tjeng, Phys. Rev. Lett. 97, 116402 (2006).

${ }^{47}$ See, e.g., G. Panaccione, F. Offi, M. Sacchi, and P. Torelli, C. R. Physique 9, 524 (2008); K. Kobayashi, Nucl. Instrum. Methods A 601, 32 (2009)

${ }^{48}$ B. McWhan, A. Menth, J. P. Remeika, W. F. Brinkman, and T. M. Rice, Phys. Rev. B 7, 1920 (1973).

${ }^{49}$ S. Y. Ezhov, V. I. Anisimov, D. I. Khomskii, and G. A. Sawatzky, Phys. Rev. Lett. 83, 4136 (1999). 
${ }^{50}$ S. Kobayashi, Y. Nohara, S. Yamamoto, and T. Fujiwara, Phys. Rev. B 78, 155112 (2008).

${ }^{51}$ G. Panaccione, M. Altarelli, A. Fondacaro, A. Georges, S. Huotari, P. Lacovig, A. Lichtenstein, P. Metcalf, G. Monaco, F. Offi, L. Paolasini, A. Poteryaev, M. Sacchi, and O. Tjernberg, Phys. Rev. Lett. 97, 116401 (2006).

${ }^{52}$ S.-K. Mo, J. D. Denlinger, H.-D. Kim, J.-H. Park, J. W. Allen, A. Sekiyama, A. Yamasaki, K. Kadono, S. Suga, Y. Saitoh, T. Muro, P. Metcalf, G. Keller, K. Held, V. Eyert, V. I. Anisimov, and D. Vollhardt, Phys. Rev. Lett. 90, 186403 (2003).

${ }^{53}$ G. Keller, K. Held, V. Eyert, D. Vollhardt, and V. I. Anisimov, Phys. Rev. B 70, 205116 (2004).

${ }^{54}$ A. I. Poteryaev, J. M. Tomczak, S. Biermann, A. Georges, A. I. Lichtenstein, A. N. Rubtsov, T. Saha-Dasgupta, and O. K. Andersen, Phys. Rev. B 76, 085127 (2007).

${ }^{55}$ E. Papalazarou, M. Gatti, M. Marsi, V. Brouet, F. Iori, L. Reining, E. Annese, I. Vobornik, F. Offi, A. Fondacaro, S. Huotari, P. Lacovig, O. Tjernberg, N. B. Brookes, M. Sacchi, P. Metcalf, and G. Panaccione, Phys. Rev. B 80, 155115 (2009).

${ }^{56}$ P. D. Dernier, J. Phys. Chem. Solids 31, 2569 (1970).

${ }^{57}$ P. D. Dernier and M. Marezio, Phys. Rev. B 2, 3771 (1970).

${ }^{58}$ R. M. Moon, Phys. Rev. Lett. 25, 527 (1970).

${ }^{59}$ G. A. Thomas, D. H. Rapkine, S. A. Carter, A. J. Millis, T. F. Rosenbaum, P. Metcalf, and J. M. Honig, Phys. Rev. Lett. 73, 1529 (1994).

${ }^{60}$ H. Fujiwara, A. Sekiyama, S.-K. Mo, J. W. Allen, J. Yamaguchi, G. Funabashi, S. Imada, P. Metcalf, A. Higashiya, M. Yabashi, K. Tamasaku, T. Ishikawa, and S. Suga, Phys. Rev. B 84, 075117 (2011).

${ }^{61}$ J. M. Honig and T. B. Reed, Phys. Rev. 174, 1020 (1968).

${ }^{62}$ R. M. Moon, T. Riste, W. C. Koehler, and S. C. Abrahams, J. Appl. Phys. 40, 1445 (1969).

${ }^{63}$ C. E. Rice and W. R. Robinson, Acta Crystallogr. Sect. B 33, 1342 (1977).

${ }^{64}$ L. F. Mattheis, J. Phys.: Condens. Matter 8, 5987 (1996).

${ }^{65}$ V. Eyert, U. Schwingenschögl, and U. Eckern, Europhys. Lett. 70, 782 (2005).

${ }^{66}$ A. I. Poteryaev, A. I. Lichtenstein, and G. Kotliar, Phys. Rev. Lett. 93, 086401 (2004).

${ }^{67}$ T. C. Koethe, Ph. D. thesis, Universität zu Köln, Germany, 2006 [http://kups.ub.uni-koeln.de/1929].

${ }^{68}$ S. H. Shin, G. V. Chandrashekhar, R. E. Loehman, and J. M. Honig, Phys. Rev. B 8, 1364 (1973).

${ }^{69}$ M. Catti, G. Sandrone, and R. Dovesi, Phys. Rev. B 55, 16122 (1997).
${ }^{70}$ A. Fujimori, I. Hase, H. Namatame, Y. Fujishima, Y. Tokura, H. Eisaki, S. Uchida, K. Takegahara, and F. M. F. de Groot, Phys. Rev. Lett. 69, 1796 (1992).

${ }^{71}$ I. Solovyev, N. Hamada, and K. Terakura, Phys. Rev. B 53, 7158 (1996).

${ }^{72}$ H. Sawada and K. Terakura, Phys. Rev. B 58, 6831 (1998).

${ }^{73}$ S. Okatov, A. Poteryaev, and A. Lichtenstein, Europhys. Lett. 70, 499 (2005).

${ }^{74}$ E. Pavarini, S. Biermann, A. Poteryaev, A. I. Lichtenstein, A. Georges, and O. K. Andersen, Phys. Rev. Lett. 92, 176403 (2004).

${ }^{75}$ L. Craco, S. Leoni, and E. Müller-Hartmann, Phys. Rev. B 74, 155128 (2006).

${ }^{76}$ L. Craco, S. Leoni, M. S. Laad, and H. Rosner, Phys. Rev. B 76, 115128 (2007)

${ }^{77}$ M. Cwik, T. Lorenz, J. Baier, R. Müller, G. André, F. Bourée, F. Lichtenberg, A. Freimuth, R. Schmitz, E. Müller-Hartmann, and M. Braden, Phys. Rev. B 68, 060401(R) (2003).

${ }^{78}$ W. Knafo, C. Meingast, A. V. Boris, P. Popovich, N. N. Kovaleva, P. Yordanov, A. Maljuk, R. K. Kremer, H. V. Löhneysen, and B. Keimer, Phys. Rev. B 79, 054431 (2009).

${ }^{79}$ G. Khaliullin and S. Maekawa, Phys. Rev. Lett. 85, 3950 (2000).

${ }^{80}$ M. W. Haverkort, Z. Hu, A. Tanaka, G. Ghiringhelli, H. Roth, M. Cwik, T. Lorenz, C. Schüßler-Langeheine, S. V. Streltsov, A. S. Mylnikova, V. I. Anisimov, C. de Nadai, N. B. Brookes, H. H. Hsieh, H.-J. Lin, C. T. Chen, T. Mizokawa, Y. Taguchi, Y. Tokura, D. I. Khomskii, and L. H. Tjeng, Phys. Rev. Lett. 94, 056401 (2005).

${ }^{81}$ M. Mochizuki and M. Imada, New J. Phys. 6, 154 (2004).

${ }^{82}$ A. C. Komarek, H. Roth, M. Cwik, W.-D. Stein, J. Baier, M. Kriener, F. Bourée, T. Lorenz, and M. Braden, Phys. Rev. B 75, 224402 (2007).

${ }^{83}$ H. Roth, Ph. D. thesis, Universität zu Köln, Germany, 2008, [http://kups.ub.uni-koeln.de/2335].

${ }^{84}$ Y. Okimoto, T. Katsufuji, Y. Okada, T. Arima, and Y. Tokura, Phys. Rev. B 51, 9581 (1995).

${ }^{85}$ I. Loa, X. Wang, K. Syassen, H. Roth, T. Lorenz, M. Hanand, and Y.-L. Mathis, J. Phys.: Condens. Matter 19, 406223 (2007).

${ }^{86}$ N. Mott, Metal-Insulator Transitions, 2nd ed. (Taylor \& Francis, London, 1990), pp. 138-139.

${ }^{87}$ D. Hughes, M. Däne, A. Ernst, W. Hergert, M. Lüders, J. B. Staunton, Z. Szotek, and W. M. Temmerman, New J. Phys. 10, 063010 (2008).

${ }^{88}$ A. Filippetti, C. D. Pemmaraju, S. Sanvito, P. Delugas, D. Puggioni, and V. Fiorentini, Phys. Rev. B 84, 195127 (2011).

${ }^{89}$ F. Fuchs, J. Furthmüller, F. Bechstedt, M. Shishkin, and G. Kresse, Phys. Rev. B 76, 115109 (2007). 